\title{
RSV infection modulates IL-15 production and MICA levels in respiratory epithelial
} cells

\author{
M.T. Zdrenghea*,\#, A.G. Telcian*, V. Laza-Stanca*, C.M. Bellettato*, , M.R. Edwards*, \\ A. Nikonova*, ${ }^{\star,}$ M.R. Khaitov*, ${ }^{*,}$ N. Azimi ${ }^{\S}$, V. Groh ${ }^{\dagger}$, P. Mallia*, \\ S.L. Johnston* and L.A. Stanciu*
}

ABSTRACT: The cytokine interleukin (IL)-15, major histocompatibility complex (MHC) class I molecules and MHC class I chain-related proteins (MIC) A and B are involved in cellular immune responses to virus infections but their role in respiratory syncytial virus (RSV) infection has not been studied. We aimed to determine how RSV infection modulates IL-15 production, MHC class I and MICA expression in respiratory epithelial cells, the molecular pathways implicated in virusinduced IL-15 production and how interferon (IFN)- $\gamma$ alters RSV-induced IL-15 production and MHC class I and MICA expression.

We infected respiratory epithelial cell lines (A549 and BEAS-2B cells) and primary bronchial epithelial cells with RSV and measured production of IL-15, expression of MHC I and MICA and the role of the transcription factor nuclear factor (NF)-кB.

We report here that RSV increases IL-15 in respiratory epithelial cells via virus replication and NF-kB-dependent mechanisms. Furthermore, RSV infection of epithelial cells upregulated cell surface expression of MICA and levels of soluble MICA. IFN- $\gamma$ upregulated RSV induction of soluble IL-15 but inhibited induction of MICA.

Upregulation of IL-15, MHC I and MICA are likely to be important mechanisms in activating immune responses to RSV by epithelial cells.

KEYWORDS: Epithelial cell, immune response, respiratory viruses

$\mathbf{R}$ espiratory syncytial virus (RSV) is a common cause of severe respiratory tract diseases in infants, children and the elderly. RSV infection of respiratory epithelial cells modulates surface and soluble molecules involved in interferon (IFN)- $\gamma$ production and cytotoxic activity of natural killer (NK) and T-cells [1-3]. The presence of a virus-inducible region in the interleukin (IL)-15 promoter [4] suggests that IL-15 is an important component of host antiviral defence mechanisms, via induction of the IFN-related transcription factors nuclear factor (NF)- $\mathrm{kB}$, IFN regulatory factors and type 1 IFNs, synthesis of type 1 effector molecules (IFN- $\gamma$, granzyme and perforin) and survival and activation of NK and effector/memory CD8+ T-cells [5-7]. IL-15 also upregulates major histocompatibility complex (MHC) class I chain-related proteins (MIC) A and B on intestinal epithelial cells [8]. Surface MIC molecules (encoded by genes within the human MHC and genetically linked to MHC class I human leukocyte antigen (HLA)-B molecules) regulate innate immunity by nonspecifically activating NKG2D+ NK and CD8+ T-cells $[9,10]$.
Therefore, IL-15 is likely to play a key role in the immune response to RSV infection but no data are available regarding RSV modulation of IL-15 production and MICA/B expression in human respiratory epithelial cells.

We aimed to determine how RSV infection modulates IL-15 production in respiratory epithelial cells and the molecular pathways implicated in virus-induced IL-15 production. In addition, because RSV infection induces a type 1 immune response characterised by increased IFN- $\gamma$ production [11], we determined how IFN- $\gamma$ alters RSVinduced IL-15 production and the levels of MHC class I and MICA molecules in epithelial cells.

\section{MATERIAL AND METHODS}

\section{Cell culture and RSV infection of respiratory} epithelial cells

RSV A2 (American Type Culture Collection, Rockville, MD, USA) was grown in Hep-2 cells and virus titre determined by plaque assay. In order to assess the specificity of live RSV-mediated

\section{AFFILIATIONS}

*Dept of Respiratory Medicine, National Heart and Lung Institute, MRC and Asthma UK Centre in Allergic Mechanisms of Asthma and Centre for Respiratory Infection, Imperial College London, London, UK.

\#Dept of Haematology, Oncology Institute, Cluj-Napoca, Romania. -Dept of Paediatrics, Centre for Rare Diseases, University of Padua, Padua, Italy.

${ }^{+} \mathrm{NRC}$ Institute of Immunology FMBA, Moscow, Russia.

${ }^{\S}$ Bioniz, LLC, Lake Forest, CA, and ${ }^{f}$ Fred Hutchinson Cancer Research Center, Seattle, WA, USA.

CORRESPONDENCE

L.A. Stanciu

Dept of Respiratory Medicine

National Heart and Lung Institute

Imperial College London

Norfolk Place

London W2 1 PG

UK

E-mail: I.stanciu@imperial.ac.uk

Received:

June 102011

Accepted after revision:

July 282011

First published online:

Aug 182011

European Respiratory Journal Print ISSN 0903-1936 Online ISSN 1399-3003 
a)


b)

BEAS-2B

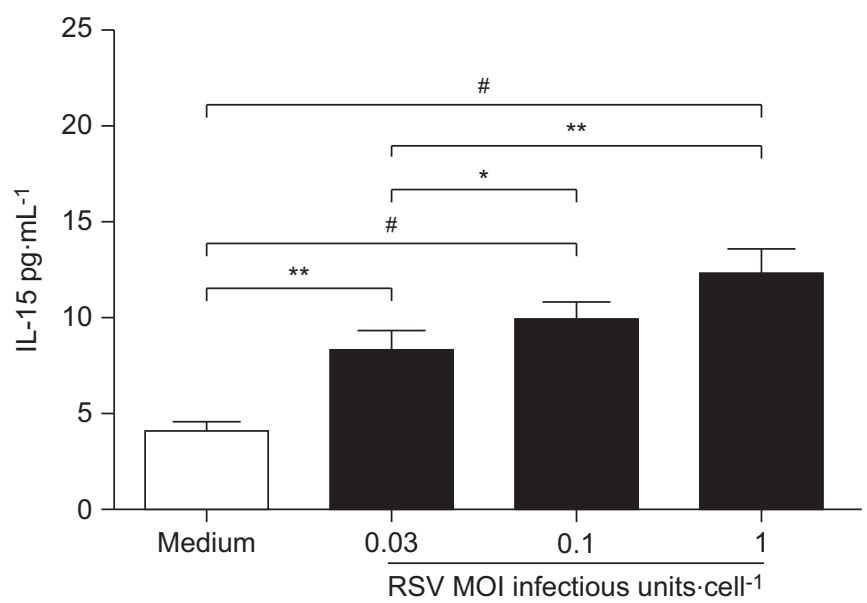

d)

BEAS-2B



FIGURE 1. Respiratory syncytial virus (RSV) increases interleukin (IL)-15 production in a dose- and time-dependent manner in respiratory epithelial cells. a) A549 cells and b) BEAS-2B cells were infected with RSV at different multiplicity of infection (MOI) and cultured for 48 and $72 \mathrm{~h}$, respectively, then supernatants were collected and IL-15 levels were measured by ELISA. c) A549 cells and d) BEAS-2B cells were infected with RSV, supernatants collected at subsequent time-points (6-72 h) and IL-15 levels measured by ELISA. e) Human primary bronchial epithelial cells (HPBEC) were infected with RSV, supernatants collected at $24 \mathrm{~h}$ and IL-15 levels measured by ELISA. All results are shown as mean \pm SEM, $n=3-6 .{ }^{*}: p<0.05 ;{ }^{*}: p<0.01 ;{ }^{*}: p<0.005$. 
a)

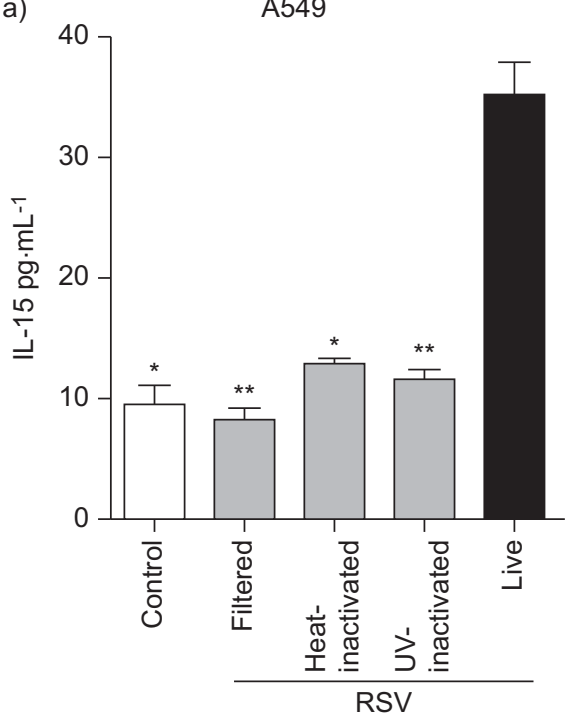

b)

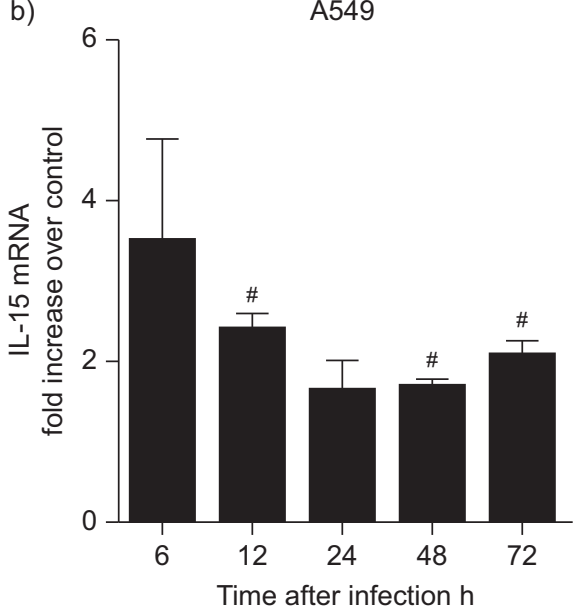

c)

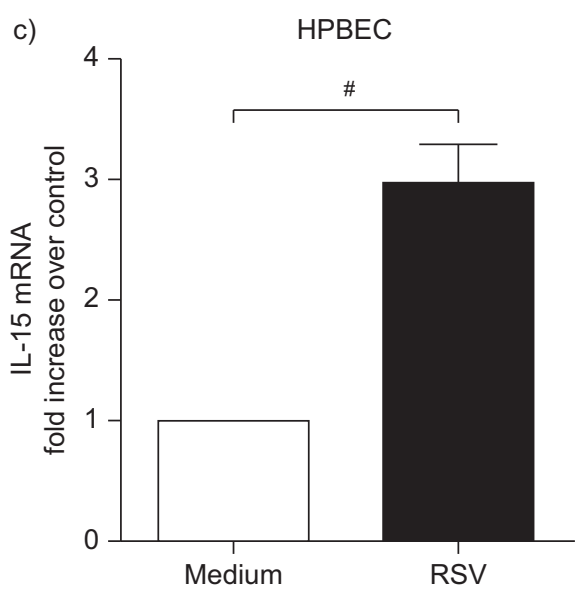

FIGURE 2. Respiratory syncytial virus (RSV) induces interleukin (IL)-15 via virus replication and by increasing gene expression. a) Inactivation of RSV abolished IL-15 induction in A549 cells at $48 \mathrm{~h}(\mathrm{n}=3)$. b) A549 cells $(n=3)$ and c) human primary bronchial epithelial cells (HPBEC; $n=6)$ were infected with RSV, total RNA was extracted from cell lysates b) after 6-72 $\mathrm{h}$ and c) after $24 \mathrm{~h}$, and IL-15 mRNA measured by quantitative RT-PCR. Results are presented as fold increase of the mRNA copy numbers in infected cells over noninfected control. All data are mean \pm SEM. *: $p<0.05$ versus live RSV; **: $p<0.01$ versus live RSV; *: $p<0.05$ versus noninfected control.

responses, UV-inactivated, thermal-inactivated and filtered RSV were used as controls [2, 3]. Bronchial (BEAS-2B) and alveolar (A549) epithelial cell lines (European Collection of Cell Cultures, Salisbury, UK) and human primary bronchial epithelial cells (HPBEC) (Clonetics, Cambrex BioScience, Walkersville, MD, USA) were cultured with RSV as previously described [2, 3]. Semiconfluent cell monolayers were exposed to RSV at different multiplicity of infection (MOI; infectious units $\cdot \mathrm{cell}^{-1}$ ) or inactivated RSV for $1 \mathrm{~h}$ with gentle shaking, the virus was washed and fresh medium with or without IFN- $\gamma\left(50 \mathrm{ng} \cdot \mathrm{mL}^{-1}\right.$; R\&D Systems Ltd, Abingdon, UK) was added. Time zero was considered to be the moment when virus was removed. Epithelial cells were harvested for flow cytometry and supernatants, RNA or protein lysates were stored at $-80^{\circ} \mathrm{C}$.

\section{Flow cytometry}

Epithelial cells were harvested at different time-points and processed as described elsewhere [2, 3]. Surface MHC class I and MICA were detected on respiratory epithelial cells by direct staining with mouse antihuman monoclonal antibodies: phycoerythrin (PE)-labelled human MICA (R\&D Systems Ltd) and allophycocyanin-labelled human HLA class I (BD Bioscience, Oxford, UK).

We acquired at least 10,000 events using a BD LSR flow cytometer (BD Bioscience). Results were expressed as mean fluorescent intensity (MFI) after subtracting the MFI of the control cells stained with the appropriate isotype control antibodies. Stimulation experiments were expressed as fold increase of MFI of experimental condition over medium-treated cells of at least three separate experiments.

\section{ELISA for IL-15 and soluble MICA}

Levels of IL-15 were measured in culture supernatants by ELISA using commercially available matched-pair antibodies (R\&D Systems Ltd) and recombinant IL-15 (Biosource UK Ltd, London, UK).
The lower limit of sensitivity of the assay was $0.25 \mathrm{pg} \cdot \mathrm{mL}^{-1}[12]$. Levels of soluble MICA were determined with Human MICA ELISA development kit, DuoSet (R\&D Systems Ltd; sensitivity $\left.31.25 \mathrm{pg} \cdot \mathrm{mL}^{-1}\right)$.

\section{RNA extraction, reverse transcription and real-time PCR}

Total RNA was extracted with RNeasy Mini Kit (Qiagen Ltd, Crawley, UK) and $2 \mu \mathrm{g}$ used for cDNA synthesis (Omniscript RT Kit; Qiagen Ltd). Quantitative RT-PCR (TaqMan; Qiagen Ltd) was performed using specific primers and probe for IL-15 designed by the authors [12].

Data were analysed using version 1.4, ABI Prism 7000 SDS software (Applied Biosystems, Warrington, UK). IL-15 gene expression levels were normalised to 18S rRNA (used as endogenous loading control) and presented as fold increase relative to medium.

\section{NF- $\boldsymbol{\kappa} \boldsymbol{B}$ activation}

The effect of NF- $\kappa$ B activation on RSV-induced IL-15 production was evaluated using an IKB kinase (IKK)2 inhibitor (AS602868) as previously described [13]. AS602868 inhibits the activation of the IKK kinase responsible for the degradation of NF- $\mathrm{KB}$ inhibitors, and consequently decreases intracellular levels of NF- $\kappa B$ [13]. We used the IKK2 inhibitor AS602868 at a concentration previously shown to be optimal for rhinovirus-induced tumour necrosis factor- $\alpha$ inhibition without cell toxicity [13].

\section{Functional analysis of IL-15 promoter activity}

Plasmids containing the full-length IL-15 promoter with secreted alkaline phosphatase (seAP) reporter (hIL-15-seAP), plasmids containing deletions of the IL-15-seAP and the NF- $\mathrm{BB}$ mutant plasmids (mtNF-кB-897-seAP) were gifts from N. Azimi [14].

To determine the regions of the IL-15 promoter at which RSV upregulation occurs, the plasmids coupled to seAP reporter were 
a)
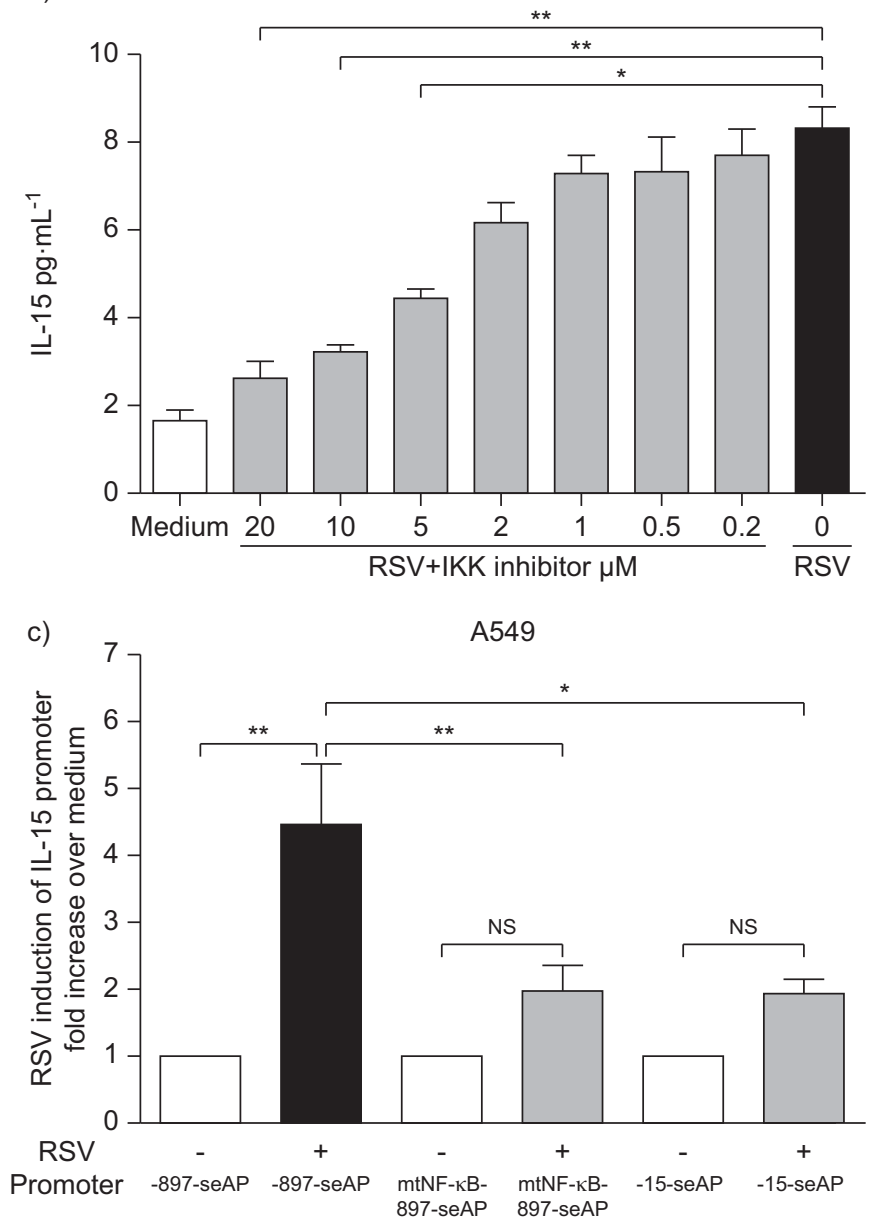

b)

HPBEC

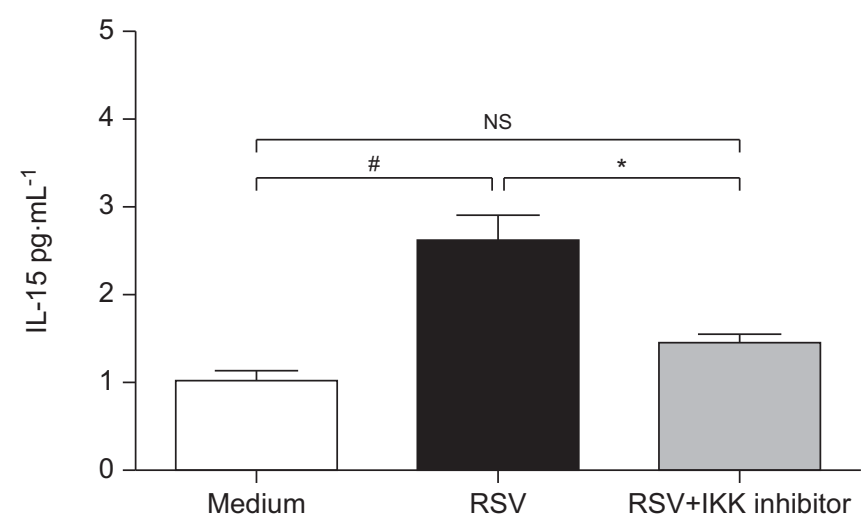

FIGURE 3. Respiratory syncytial virus (RSV) increases interleukin (IL)-15 promoter activation via nuclear factor (NF)-kB activation. a) A549 cells cultured for $8 \mathrm{~h}$ in the presence of increasing concentrations of IKB kinase (IKK) inhibitor AS602868 were infected with RSV for $1 \mathrm{~h}$, washed and the IKK inhibitor added again. The culture supernatants were collected at $14 \mathrm{~h}$ post-infection and IL-15 levels measured by ELISA. b) Human primary bronchial epithelial cells (HPBEC) treated with $10 \mu \mathrm{M}$ AS602868 were infected with RSV, culture supernatants were collected at $24 \mathrm{~h}$ post-infection and IL-15 levels measured by ELISA. c) A549 cells transfected with plasmids containing secreted alkaline phosphatase (seAP) reporter construct of intact IL-15 promoter (-897-seAP), plasmids with deletion of almost the entire IL-15 promoter (-15-seAP) and plasmids containing reporter construct of IL-15 promoter with NF-kB ligation site mutated (mtNF-kB-897-seAP) were infected with RSV and supernatants collected at $24 \mathrm{~h}$. seAP activity was measured and results expressed as a ratio between RSV-infected cells and uninfected cells in medium. All RSV infections were multiplicity of infection 1 infectious unit.cell ${ }^{-1}$. Data are mean \pm SEM, $n=3$. *: $p<0.05$; **: $p<0.01 ; *: p<0.005$; NS: nonsignificant.

transfected into uninfected or RSV-infected A549 cells using electroporation by the calcium phosphate method (CalPhos mammalian transfection kit; BD Biosciences Clontech Laboratories, Palo Alto, CA, USA). At different time-points, the supernatants were collected, protein content measured (BioRad Protein Assay, Bradford method; BioRad Laboratories Ltd, Hemel Hempstead, UK), and equivalent amounts assayed for reporter activity by standard seAP assay (SEAP reporter gene assay; Roche Diagnostics Ltd, Burgess Hill, UK). Results were normalised for transfection efficiency using co-transfection of a $\beta$ galactoside reporter construct. The promoter activity was expressed as the fold induction compared with that of the negative control.

\section{Statistical analysis}

The results were analysed using GraphPad Prism version 4.00 for Windows (GraphPad Software, San Diego, CA, USA). Results of at least three separate experiments were expressed as mean \pm SEM.
ANOVA followed where appropriate by paired t-test was used for the comparisons between different experimental conditions. Values of $\mathrm{p}<0.05$ were considered statistically significant.

\section{RESULTS}

\section{RSV upregulates IL-15 production in epithelial cells}

Alveolar A549 and bronchial BEAS-2B cells constitutively express low levels of IL-15 and infection by RSV significantly increased levels of IL-15 in A549 (fig. 1a) and BEAS-2B (fig. 1b) cell supernatants in a concentration- and time-dependent manner up to $72 \mathrm{~h}$ post-infection (fig. 1c and d). RSV infection also increased IL-15 production in HPBEC (fig. 1e).

To establish whether virus replication was required for induction of IL-15, we next determined IL-15 protein release in supernatants of RSV-infected respiratory epithelial cells and in cells treated with nonreplicative virus (UV- or heat-inactivated) or inocula from which virus had been removed by molecular weight 
a)



b)

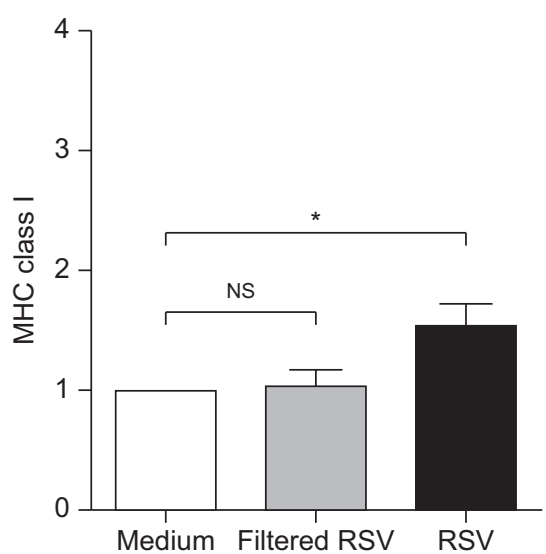

c)

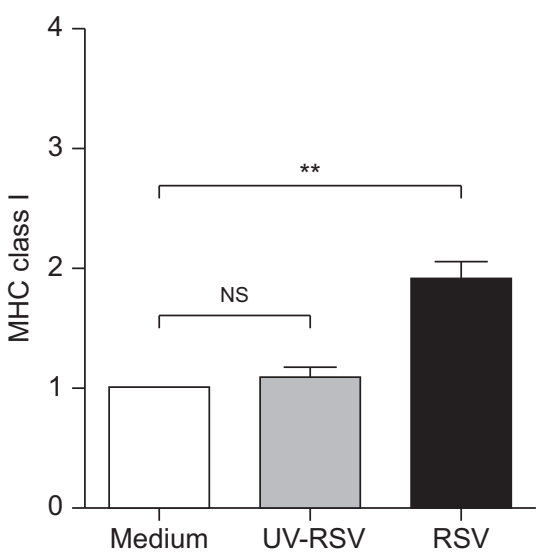

FIGURE 4. Respiratory syncytial virus (RSV) replication increases major histocompatibility complex (MHC) class I levels. a) A549, b) BEAS-2B cells and c) human primary bronchial epithelial cells (HPBEC) were cultured with RSV multiplicity of infection 1 infectious unit.cell ${ }^{-1}$, UV-inactivated RSV or filtered RSV for 24 h, cells harvested and human leukocyte antigen class I surface molecules determined by flow cytometry. Data are mean \pm SEM mean fluorescent intensity fold increase over medium, $\mathrm{n}=3$. *: $p<0.05 ; * *: p<0.01 ;$ NS: nonsignificant.

filtration. RSV significantly induced increased levels of IL-15 $48 \mathrm{~h}$ post-infection compared with A549 epithelial cells treated with UV- or heat-inactivated virus, filtered virus or medium only (fig. 2a), indicating that IL-15 induction in bronchial epithelial cells was due to live virus active replication and not to soluble factors in the virus inoculum.

\section{RSV increases IL-15 gene expression and promoter activation in epithelial cells and IL-15 induction is NF- $\kappa B$ dependent}

In order to investigate whether RSV increases IL-15 protein by a pre-transcriptional mechanism via upregulation of mRNA levels or by increasing the release of constitutive intracellular protein, we measured IL-15 mRNA expression. In RSV-infected A549 cells, IL-15 mRNA levels were increased at 12, 48 and $72 \mathrm{~h}$ postinfection compared with the control (fig. 2b). IL-15 mRNA also increased in HPBEC following RSV infection at $24 \mathrm{~h}$ (fig. 2c).

As IL-15 induction in other systems has been reported to be NF$\kappa \mathrm{B}$ dependent [15], we next investigated the role of NF- $\kappa \mathrm{B}$ in RSVinduced IL-15 production in epithelial cells. RSV-induced IL-15 expression in A549 cells was significantly decreased in a concentration-dependent manner upon addition of the pharmacological IKK2 inhibitor AS602868 (fig. 3a), and induction in HPBEC was also significantly inhibited by IKK2 inhibition (fig. 3b). These data suggest that RSV induction of IL-15 is dependent on IKK2 and probably involves the canonical NF- $\kappa B$ pathway.

In order to investigate whether RSV induction of IL-15 involved $\mathrm{IL}-15$ promoter activation, and to confirm the role of NF- $\mathrm{\kappa B}$ in activation of the IL-15 promoter by RSV, we used plasmids containing full-length IL-15 promoter (-897-seAP), plasmids with IL-15 promoter almost absent (-15-seAP) and full-length promoter plasmids containing mutations at the -75/-65 NF-kB-binding site (mtNF-кB-897-seAP). RSV infection induced activation of the full-length IL-15 promoter construct (fold increase 4.45 \pm 0.92 ; $\mathrm{p}<0.01$ ) when compared with uninfected transfected cells. Cells transfected with the promoters -15-seAP and mtNF-кB-897-seAP did not significantly increase expression of IL-15 promoter when infected with RSV (fig. 3c). These data provide evidence that RSV induction of IL-15 involves IL-15 promoter activation and further evidence that the NF- $\mathrm{BB}$ ligation site is necessary for the activation of IL-15 promoter by RSV.

\section{RSV and surface MHC class I and MICA/MICB molecules}

Because MHC class I molecules are related to MIC molecules, we investigated how RSV modulates these molecules. MHC class I molecules have an important role in innate immunity (by controlling NK cell activity) and, via antigen-presentation, in acquired antiviral immunity.

RSV infection increased surface levels of MHC class I molecules in A549, BEAS-2B and HPBEC $(p<0.05, p<0.05$ and $p<0.01$, respectively) (fig. 4 ) at $24 \mathrm{~h}$ in a replication-dependent, doseresponse and time-dependent manner (data not shown).

We were interested to determine how RSV modulates MICA and MICB molecules. It was reported that surface MICA molecules enhance nonspecific activation of NKG2D+ NK cells and CD8+ Tcells and that in some circumstances released soluble forms of MICA inhibit the immune response [16].

Using an anti-MIC antibody (recognising both MICA and MICB molecules; 6D4.6 antibody; gift from V. Groh) [17], we found that HPBEC, A549 and BEAS-2B cells constitutively expressed MICA/MICB molecules (representative histogram in HPBEC shown in fig. 5a), as previously reported [18, 19].

Using a specific MICA antibody that became commercially available during the present study, we found that RSV infection increased surface levels of MICA in BEAS-2B cells in a time- and dose-dependent manner (fig. $5 \mathrm{~b}$ and c, respectively). RSV infection also increased levels of soluble MICA levels produced by BEAS-2B cells in a dose- and time-dependent manner (fig. 5d).

\section{IFN- $\gamma$ increases RSV-induced IL-15 levels but downregulates RSV induction of MICA}

It has been reported that IFN- $\gamma$ increases IL-15 levels in bronchial epithelial cells from lung cancer patients and in the BEAS-2B cell line $[20,21]$. Therefore, we investigated whether IFN- $\gamma$ influenced 


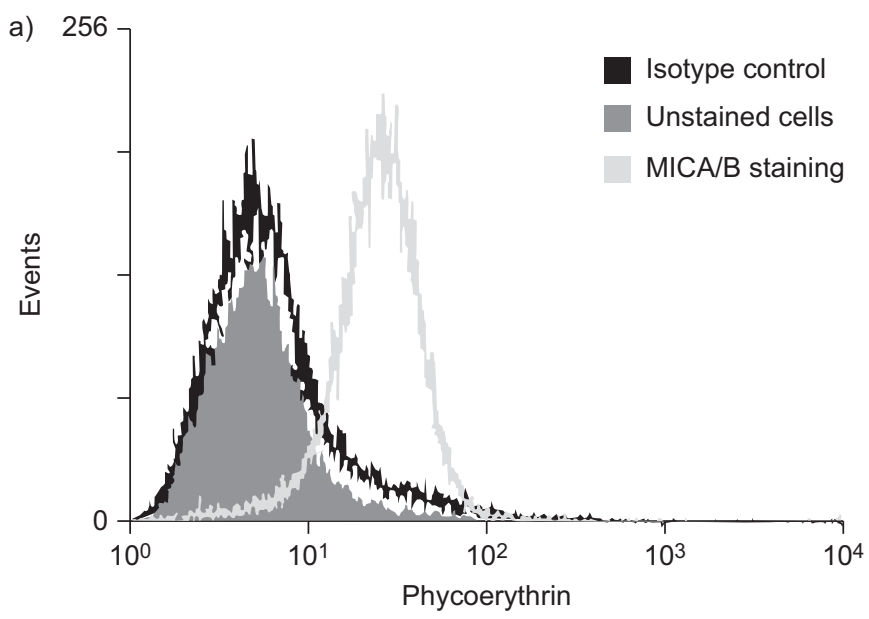

c)

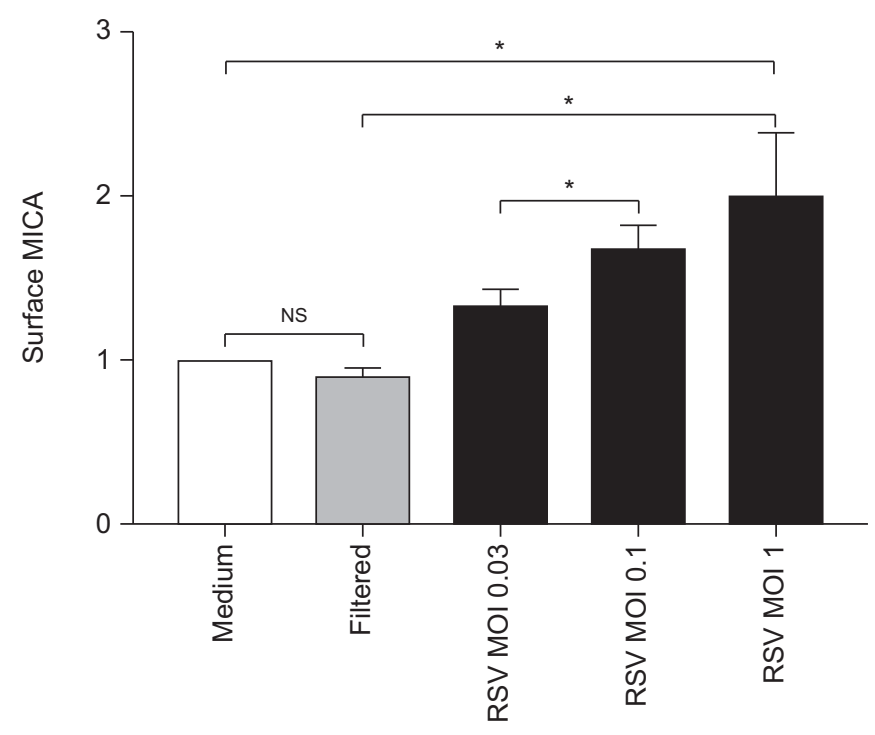

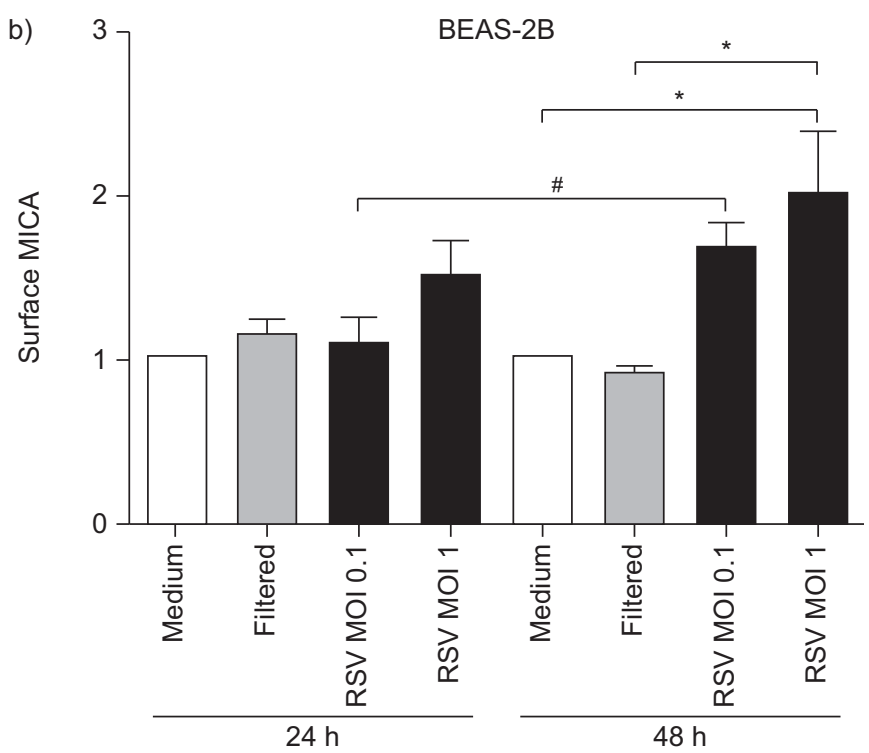

d)

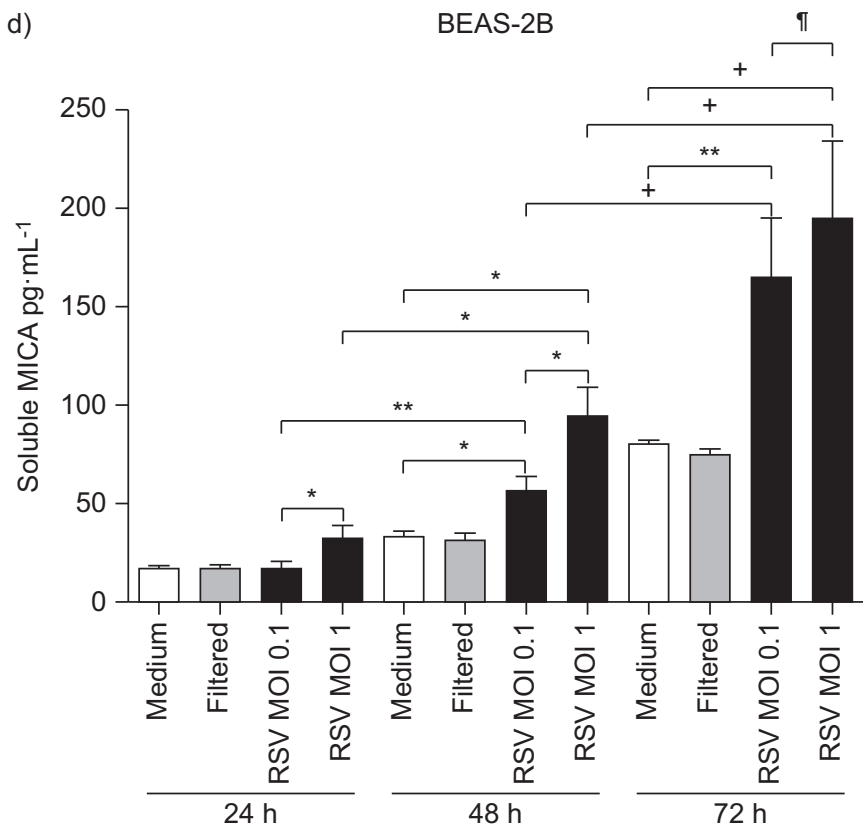

FIGURE 5. Respiratory syncytial virus (RSV) induces surface and soluble expression of major histocompatibility complex class I chain-related protein (MIC) A in respiratory epithelial cells. a) Human primary bronchial epithelial cells (HPBEC) constitutively express surface MICA/B molecules. Data are from one representative experiment out of three. $\mathrm{b}$ and c) BEAS-2B cells were cultured with medium, filtered RSV and RSV multiplicity of infection (MOI) $0.03,0.1$ and 1 infectious unit·cell ${ }^{-1}$ for up to $72 \mathrm{~h}$. Cells were harvested at b) 24 and $48 \mathrm{~h}$ and c) $48 \mathrm{~h}$ to determine surface MICA by flow cytometry. Data are mean fluorescent intensity fold increase over medium. d) Supernatants were harvested at 24,48 and $72 \mathrm{~h}$ to determine soluble MICA by ELISA. Data are mean $\pm \mathrm{SEM}, \mathrm{n}=3 .{ }^{*}: p<0.05 ;{ }^{*}: p<0.01 ;{ }^{*}: p=0.08 ;{ }^{\circ}: p=0.06 ;{ }^{+}: p<0.005$.

IL-15 levels, MHC class I expression or surface or soluble levels of MICA, either alone, or in combination with RSV infection. The presence of IFN- $\gamma$ in the culture milieu significantly increased IL15 secretion, and enhanced RSV-induced soluble IL-15 levels in BEAS-2B cells at $24 \mathrm{~h}(\mathrm{p}<0.01)$ (fig. 6a).

Treatment of epithelial cells with IFN- $\gamma$ alone also significantly increased surface MHC class I expression on epithelial cells at $24 \mathrm{~h}(\mathrm{p}<0.01$ for BEAS-2B and $\mathrm{p}<0.001$ for HPBEC) (fig. $6 \mathrm{c}$ and $\mathrm{d}$, respectively). The presence of IFN- $\gamma$ in culture of RSV-infected cells had no effect on MHC class I in either epithelial cell line or HPBEC. In contrast, treatment with IFN- $\gamma$ significantly decreased both surface and soluble MICA in BEAS-2B cells at $24 \mathrm{~h}$ and $48 \mathrm{~h}$ (fig. 7). IFN- $\gamma$ also inhibited the RSV-induced increase in surface MICA in BEAS-2B cells at $24 \mathrm{~h}$ and blocked the release of soluble MICA. The same inhibitory effect was observed at $48 \mathrm{~h}$ (fig. 7).

\section{DISCUSSION}

We here show evidence that in vitro RSV infection upregulates airway epithelial cell IL-15 production via virus replication, activation of transcription factor NF- $\mathrm{KB}$ and upregulation of the IL-15 gene. RSV infection also upregulated surface MHC class I molecules and surface and soluble MICA molecules. RSV 

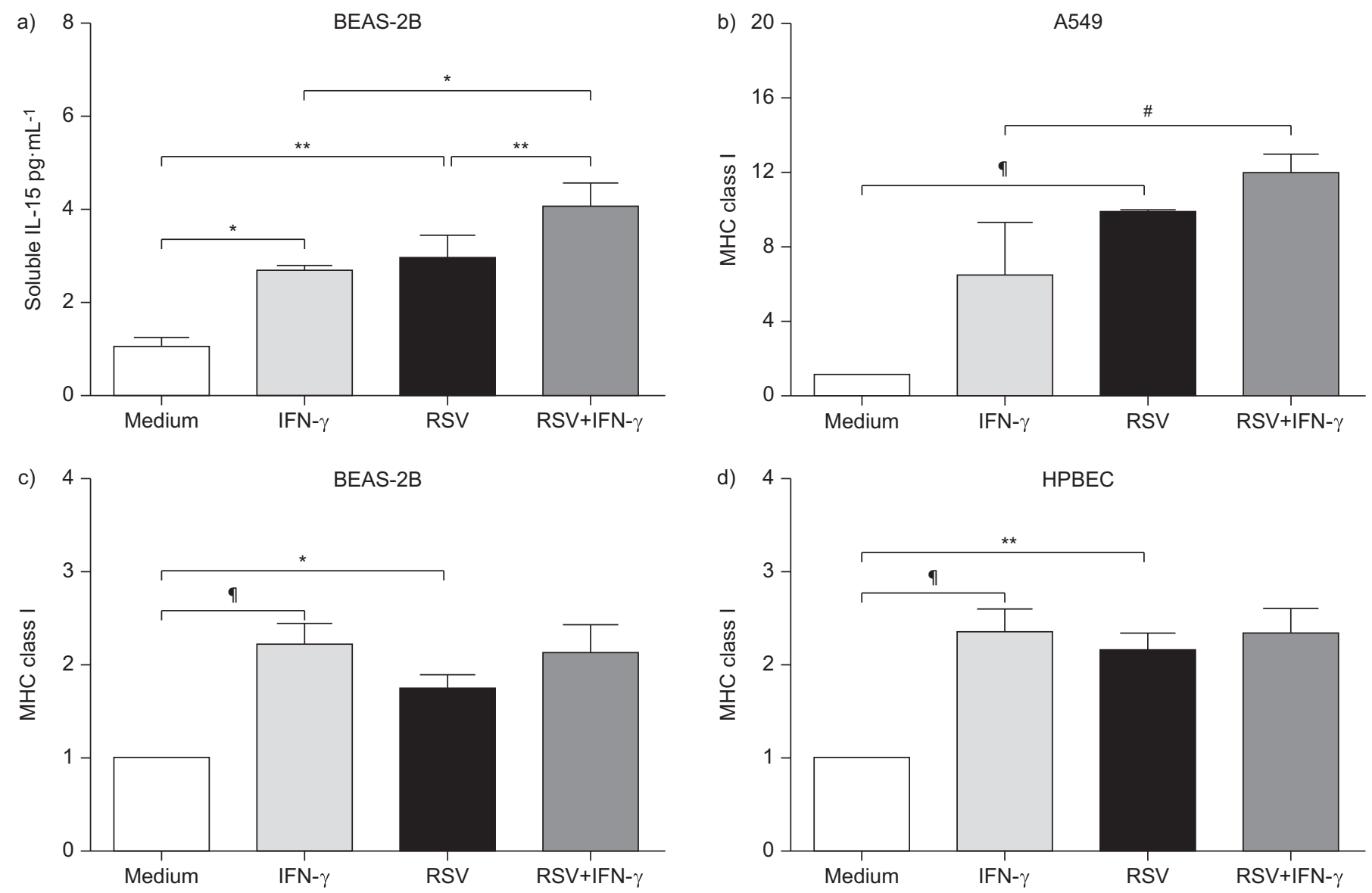

FIGURE 6. Interferon (IFN)- $\gamma$ increases respiratory syncytial virus (RSV)-induced interleukin (IL)-15 and surface major histocompatibility complex (MHC) class I levels. a) BEAS-2B cells were pre-treated with IFN- $\gamma\left(50 \mathrm{ng} \cdot \mathrm{mL}^{-1}\right)$, infected or not with RSV multiplicity of infection 1 infectious unit cell ${ }^{-1}$, cultured up to $72 \mathrm{~h}$ and IL-15 measured. Data shown are mean \pm SEM from four individual experiments at 24 h. b) A549 cells $(n=3), c)$ BEAS-2B cells $(n=5)$ and d) human primary bronchial epithelial cells (HPBEC; $\mathrm{n}=5$ ) were treated with medium, IFN- $\gamma$, RSV and RSV+IFN- $\gamma$, and MHC class I surface molecules were measured by flow cytometry at $24 \mathrm{~h}$. Data are mean fluorescent intensity fold increase over medium. *: $p<0.05 ;{ }^{* *}: p<0.01$; ${ }^{*}: p=0.09 ;{ }^{\circ}: p<0.005$

infection of respiratory epithelial cells in a type 1 cytokine milieu rich in IFN- $\gamma$ increased IL-15 production and MHC class I molecules, but downregulated surface and soluble MICA levels.

IL-15 has been shown to play an essential role in the survival of memory/effector CD8+ T-cells and to activate cytolytic function independent of $\mathrm{T}$-cell receptor specificity (NK-like killing) in effector CD8+ T-cells through NKG2D [8], helping to eliminate target cells. IL-15 gene and protein expression have been previously reported in respiratory epithelial cells [22, 23] and detected in bronchial biopsies from healthy controls as well as from patients with inflammatory lung diseases [22]. RSV upregulates IL-15 mRNA in a monocyte cell line but the effect of RSV on IL-15 production in respiratory epithelial cells has not been reported. We found that RSV infection increased production of both IL-15 mRNA and protein in respiratory epithelial cell lines and primary bronchial epithelial cells. Using a small molecule IKK inhibitor and promoter constructs, we also determined that the canonical NF- $\kappa$ B pathway plays an essential role in RSV-induced IL-15 expression. Upregulation of IL-15 by respiratory epithelial cells in response to RSV infection is likely to play an essential role in activating the cellular components of the innate immune system. We have reported deficient IL-15 production in asthmatics [12] and this may be one mechanism of increased susceptibility to respiratory virus infections in asthmatics.

We also found that RSV infection of respiratory epithelial cells upregulates both MHC class I and MICA molecules. MHC class I molecules on respiratory epithelial cells have an important role in activating antigen-specific CD8+ T-cells but may inhibit NK cells with a negative impact on controlling viral replication.

Expression of MICA on epithelial cells leads to increased cytotoxicity of NKG2D+ NK and CD8+ T-cells [17] and lysis of infected cells. It has been reported that the expression of surface MIC on human tumour cells is sufficient to overcome the inhibitory effects of MHC class I expression on NK cell killing, and NKG2D+ cytotoxicity will occur despite MHC class I expression [24]. Therefore, upregulation of MICA on epithelial cells in response to RSV infection will assure the activation of local cytotoxic NKG2D+ NK and T-cells and the eradication of virus-infected cells. However, RSV infection also upregulated levels of soluble MICA produced by respiratory epithelial cells. Soluble NKG2D ligands such as MICA impair NKG2D-mediated cytotoxicity by NK and T-cells by receptor internalisation, receptor masking or degradation of surface NKG2D [10, 16]. 
a)

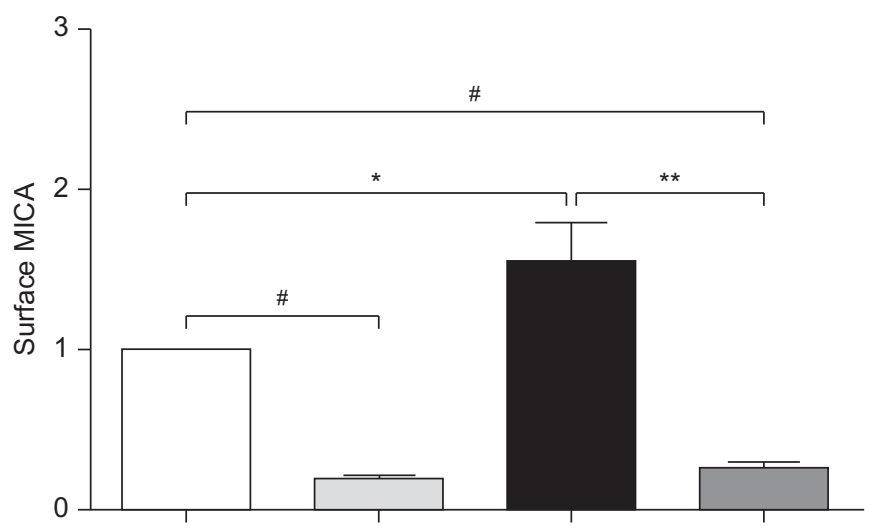

c)



b)



d)

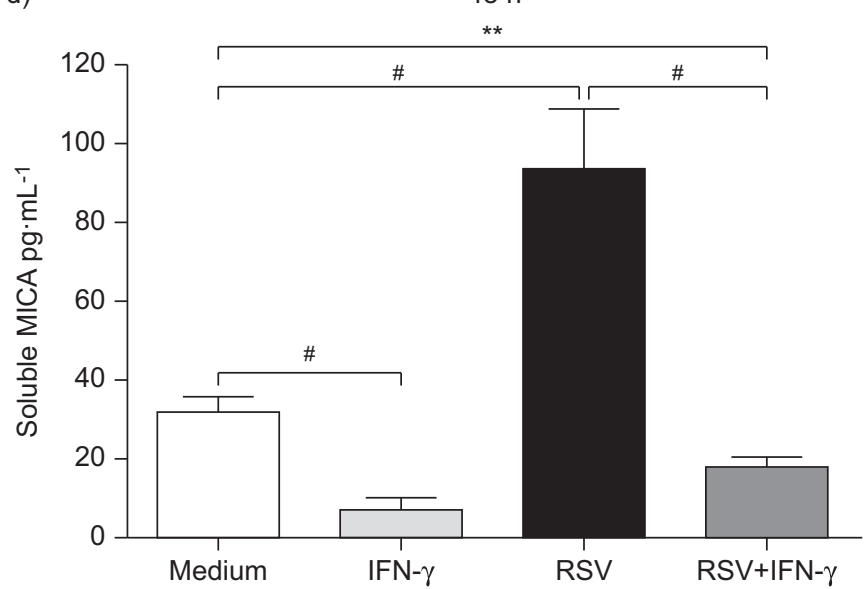

FIGURE 7. Interferon (IFN)- $\gamma$ downregulates levels of and suppresses respiratory syncytial virus (RSV) induction of surface and soluble major histocompatibility complex class I chain-related protein (MIC) A. BEAS-2B cells were treated with IFN- $\gamma$, RSV and RSV+IFN- $\gamma$. a and c) Surface MICA was measured by flow cytometry and (b and d) soluble MICA was measured by ELISA at (a and b) $24 \mathrm{~h}$ and (c and d) $48 \mathrm{~h}$. Data are mean \pm SEM, $n=3-5 .{ }^{*}: p<0.05 ;{ }^{* *}: p<0.01 ;{ }^{*}: p<0.005$.

Therefore, higher levels of soluble MICA would be expected to impair viral clearance. It is possible that upregulation of soluble MICA has a role in preventing excessive NKG2D-mediated cytotoxicity, thus averting cytolysis of bystander noninfected cells, but this hypothesis requires further investigation.

RSV induces a type 1 immune response with increased levels of IFN- $\gamma$, which, together with type I IFNs, IL-15 and co-stimulatory molecules, has an important role in virus eradication and terminating the acute infection. IFN- $\gamma$ present during in vitro RSV infection of respiratory epithelial cells inhibited the viral upregulation of surface MICA. Soluble MICA levels were also downregulated by RSV infection in the presence of IFN- $\gamma$, suggesting that the decrease in MICA surface levels by IFN- $\gamma$ was not due to proteolytic shedding of the molecules from the cell surface and that IFN- $\gamma$ probably inhibits the cell surface transport. The biological significance of IFN- $\gamma$ downregulating MICA in respiratory epithelial cells is not known. We hypothesise that airway epithelial cells infected with RSV initially upregulate surface MICA, leading to activation of cytotoxic NKG2D+ NK and CD8+ T-cells. The IFN- $\gamma$ released by these activated cells then acts as a negative feedback to downregulate MICA expression, thus terminating nonspecific activation of NKG2D+ cells, preventing excessive nonspecific immune activation and allowing the switch to antigen-specific anti-RSV adaptive immunity. This hypothesis will require further investigation in animal models of RSV infection. The effect of chronic elevated IFN- $\gamma$ levels is unknown, but, if associated with impaired upregulation of MICA in response to RSV infection, could lead to impaired viral clearance. It was recently reported that in human tumour cells, concurrently with the upregulation of surface MHC class I, IFN- $\gamma$ downregulates the levels of surface MICA $[25,26]$. Interestingly, chronic obstructive pulmonary disease (COPD) is associated with an exaggerated type 1 immune cell inflammatory infiltrate [7, 27]. Chronic RSV infection has been reported in COPD [28] and acute COPD exacerbations are associated with RSV infection [29, 30]. BORCHERS and co-workers $[18,31]$ demonstrated that NKG2D ligand expression is induced on pulmonary epithelial cells in response to oxidative stress, and protein levels of cell-associated MICA were significantly increased in COPD patients who smoked. Further investigation of the relationship between IFN- $\gamma$, surface and soluble MICA and RSV infection in COPD is warranted.

In conclusion, we report that RSV infection of respiratory epithelial cells is associated with increased production of IL-15 and upregulation of MHC class I and MICA. These are likely to be essential for an adequate immune response to RSV infection 
and dysregulation of these mechanisms may contribute to impaired viral clearance.

\section{SUPPORT STATEMENT}

This work was supported by grants from the Wellcome Trust (London, UK; grants 063717 and $083567 / Z / 07 / Z$ for the Centre for Respiratory Infection (Imperial College London) and a Wellcome Trust travel grant 063967 to M.T. Zdrenghea); the European Respiratory Society (fellowships to M.T. Zdrenghea, A.G. Telcian, C.M. Bellettato and A. Nikonova); Asthma UK (grants 02/027 and 05/067); the British Lung Foundation (grants P04/13 and P06/3) and British Lung Foundation/Severin Wunderman Family Foundation Programme Grant 00/02; the Medical Research Council (London; MRC grant G0601236); the British Medical Association (HC Roscoe Fellowships to A.G. Telcian, V. Laza-Stanca and P. Mallia); the National Institute of Health Research Biomedical Research Centre (London) funding scheme; the European Academy of Allergy and Clinical Immunology (EAACI; Zurich, Switzerland; C.M. Bellettato, M.R. Khaitov and A. Nikonova); Imperial College London; and Union Chimique Belge (UCB) Institute of Allergy fellowships (Brussels, Belgium).

\section{STATEMENT OF INTEREST}

A statement of interest for N. Azimi can be found at www.erj. ersjournals.com/site/misc/statements.xhtml

\section{REFERENCES}

1 Wang SZ, Hallsworth PG, Dowling KD, et al. Adhesion molecule expression on epithelial cells infected with respiratory syncytial virus. Eur Respir J 2000; 15: 358-366.

2 Stanciu LA, Bellettato CM, Laza-Stanca V, et al. Expression of programmed death-1 ligand (PD-L) 1, PD-L2, B7-H3, and inducible costimulator ligand on human respiratory tract epithelial cells and regulation by respiratory syncytial virus and type 1 and 2 cytokines. J Infect Dis 2006; 193: 404-412.

3 Telcian AG, Laza-Stanca V, Edwards MR, et al. RSV-induced bronchial epithelial cell PD-L1 expression inhibits CD8+ T cell nonspecific antiviral activity. J Infect Dis 2011; 203: 85-94.

4 Azimi N, Shiramizu KM, Tagaya Y, et al. Viral activation of interleukin-15 (IL-15): characterization of a virus-inducible element in the IL-15 promoter region. J Virol 2000; 74: 7338-7348.

5 Liu K, Catalfamo M, Li Y, et al. IL-15 mimics T cell receptor crosslinking in the induction of cellular proliferation, gene expression, and cytotoxicity in CD8+ memory T cells. Proc Natl Acad Sci USA 2002; 99: 6192-6197.

6 Foong YY, Jans DA, Rolph MS, et al. Interleukin-15 mediates potent antiviral responses via an interferon-dependent mechanism. Virology 2009; 393: 228-237.

7 Freeman CM, Han MK, Martinez FJ, et al. Cytotoxic potential of lung CD8+ T cells increases with chronic obstructive pulmonary disease severity and with in vitro stimulation by IL-18 or IL-15. J Immunol 2010; 184: 6504-6513.

8 Meresse B, Chen Z, Ciszewski C, et al. Coordinated induction by IL15 of a TCR-independent NKG2D signaling pathway converts CTL into lymphokine-activated killer cells in celiac disease. Immunity 2004; 21: 357-366.

9 Groh V, Bahram S, Bauer S, et al. Cell stress-regulated human major histocompatibility complex class I gene expressed in gastrointestinal epithelium. Proc Natl Acad Sci USA 1996; 93: 12445-12450.

10 Champsaur M, Lanier LL. Effect of NKG2D ligand expression on host immune responses. Immunol Rev 2010; 235: 267-285.

11 Janssen $\mathrm{R}$, Pennings J, Hodemaekers $\mathrm{H}$, et al. Host transcription profiles upon primary respiratory syncytial virus infection. J Virol 2007; 81: 5958-5967.
12 Laza-Stanca V, Message SD, Edwards MR, et al. The role of IL-15 deficiency in the pathogenesis of virus-induced asthma exacerbations. PLoS Pathog 2011; 7: e1002114.

13 Laza-Stanca V, Stanciu LA, Message SD, et al. Rhinovirus replication in human macrophages induces NF- $\kappa \mathrm{B}-$ dependent tumor necrosis factor- $\alpha$ production. J Virol 2006; 80: 8248-8258.

14 Azimi N, Brown K, Bamford RN, et al. Human T cell lymphotropic virus type I Tax protein trans-activates interleukin 15 gene transcription through an NF-kB site. Proc Natl Acad Sci USA 1998; 95: 2452-2457.

15 Ennaciri J, Ahmad R, Menezes J. Interaction of monocytic cells with respiratory syncytial virus results in activation of NF- $\kappa B$ and PKC $-\alpha / \beta$ leading to up-regulation of IL-15 gene expression. J Leukoc Biol 2007; 81: 625-631.

16 Groh V, Wu J, Yee C, et al. Tumour-derived soluble MIC ligands impair expression of NKG2D and T-cell activation. Nature 2002; 419: 734-738.

17 Groh V, Rhinehart R, Randolph-Habecker J, et al. Costimulation of CD $8 \alpha \beta$ T cells by NKG2D via engagement by MIC induced on virus-infected cells. Nat Immunol 2001; 2: 255-260.

18 Borchers MT, Harris NL, Wesselkamper SC, et al. NKG2D ligands are expressed on stressed human airway epithelial cells. Am J Physiol Lung Cell Mol Physiol 2006; 291: L222-L231.

19 Kraetzel K, Stoelcker B, Eissner G, et al. NKG2D-dependent effector function of bronchial epithelium-activated alloreactive Tcells. Eur Respir J 2008; 32: 563-570.

20 Stoeck M, Kromer W, Gekeler V. Induction of IL-15 mRNA and protein in A549 cells by pro-inflammatory cytokines. Immunobiology 1998; 199: 14-22.

21 Ge N, Nishioka Y, Nakamura Y, et al. Synthesis and secretion of interleukin-15 by freshly isolated human bronchial epithelial cells. Int Arch Allergy Immunol 2004; 135: 235-242.

22 Muro S, Taha R, Tsicopoulos A, et al. Expression of IL-15 in inflammatory pulmonary diseases. J Allergy Clin Immunol 2001; 108: $970-975$.

23 Hocke AC, Hartmann IK, Eitel J, et al. Subcellular expression pattern and role of IL-15 in pneumococci induced lung epithelial apoptosis. Histochem Cell Biol 2008; 130: 165-176.

24 Groh V, Rhinehart R, Secrist $\mathrm{H}$, et al. Broad tumor-associated expression and recognition by tumor-derived $\gamma \delta$ T cells of MICA and MICB. Proc Natl Acad Sci USA 1999; 96: 6879-6884.

25 Yadav D, Ngolab J, Lim RS, et al. Cutting edge: down-regulation of MHC class I-related chain A on tumor cells by IFN- $\gamma$-induced microRNA. J Immunol 2009; 182: 39-43.

26 Schwinn N, Vokhminova D, Sucker A, et al. Interferon- $\gamma$ downregulates NKG2D ligand expression and impairs the NKG2Dmediated cytolysis of MHC class I-deficient melanoma by natural killer cells. Int J Cancer 2009; 124: 1594-1604.

27 Hogg JC, Chu F, Utokaparch S, et al. The nature of small-airway obstruction in chronic obstructive pulmonary disease. $N$ Engl $J$ Med 2004; 350: 2645-2653.

28 Sikkel MB, Quint JK, Mallia P, et al. Respiratory syncytial virus persistence in chronic obstructive pulmonary disease. Pediatr Infect Dis J 2008; 27: S63-S70.

29 Walsh EE, Falsey AR, Hennessey PA. Respiratory syncytial and other virus infections in persons with chronic cardiopulmonary disease. Am J Respir Crit Care Med 1999; 160: 791-795.

30 Rohde G, Wiethege A, Borg I, et al. Respiratory viruses in exacerbations of chronic obstructive pulmonary disease requiring hospitalisation: a case-control study. Thorax 2003; 58: 37-42.

31 Borchers MT, Wesselkamper SC, Curull V, et al. Sustained CTL activation by murine pulmonary epithelial cells promotes the development of COPD-like disease. J Clin Invest 2009; 119: 636-649. 